\title{
Efeitos ambientais sobre características reprodutivas em ovinos Santa Inês
}

\author{
Environmental effects on reproductive traits in Santa Ines sheep
}

\author{
REGO NETO, Aurino de Araujo ${ }^{1 *}$; SARMENTO, José Lindenberg Rocha ${ }^{2}$; SANTOS, \\ Natanael Pereira da Silva ${ }^{1}$; BIAGIOTTI, Daniel ${ }^{1}$; SANTOS, Gleyson Vieira dos ${ }^{3}$; \\ SENA, Luciano Silva ${ }^{3}$; GUIMARÃES, Fabrício Fernandes ${ }^{4}$
}

\author{
${ }^{1}$ Universidade Federal do Piauí, Programa de Pós-Graduação em Ciência Animal, Teresina, Piauí, Brasil. \\ ${ }^{2}$ Universidade Federal do Piauí, Programa de Pós-Graduação em Ciência Animal e Zootecnia, Bom Jesus, \\ Piauí, Brasil. Pesquisador do CNPq. \\ ${ }^{3}$ Universidade Federal do Piauí, Programa de Pós-Graduação em Zootecnia, Bom Jesus, Piauí, Brasil. \\ ${ }^{4}$ Universidade Federal do Piauí, Bom Jesus, Piauí, Brasil. \\ *Endereço para correspondência: aurinorego@hotmail.com
}

\section{RESUMO}

O objetivo com esta pesquisa foi estudar efeitos ambientais sobre a prolificidade e a taxa de desmame em ovinos Santa Inês, por meio de modelos lineares generalizados. Foram utilizados dados do rebanho experimental de ovinos da raça Santa Inês, da Universidade Federal do Piauí, Campus Universitário Professora Cinobelina Elvas, em Bom Jesus-PI, no período de 2008 a 2010. Os efeitos ambientais estudados foram: ano de cobertura, Período de cobertura, Período de nascimento, tipo de nascimento, sexo da cria, idade da mãe ao parto, peso da mãe ao parto e peso ao nascer como covariável. Para análise estatística foi utilizado o procedimento GENMOD contido no programa SAS. Os valores médios de prolificidade e taxa de desmame foram respectivamente 1,36 e $68,18 \%$. O efeito do peso da mãe ao parto foi significativo sobre a prolificidade. Observou-se efeito significativo de Período de nascimento sobre a taxa de desmame. As correlações entre características produtivas e reprodutivas variaram de negativas a positivas de baixa magnitude. Os efeitos de peso da mãe ao parto e peso ao nascer mostraram-se importantes fontes de variação para prolificidade e taxa de desmame, o que reflete a necessidade de considerar estes efeitos em modelos de avaliação genética e fenotípica.

Palavras-chave: efeitos fixos, ovinos deslanados, período de nascimento, prolificidade

\section{SUMMARY}

In this research we aimed to study environmental effects about litter size and weaning rate in Santa Ines sheep by means of generalized linear models. We used the data from experimental sheep herd of Santa Ines, of the Federal University of Piaui, Campus Professora Cinobelina Elvas, in Bom Jesus-PI, during 2008-2010. The environmental effects studied were: mating year, mating station, birth season, birth type, offspring sex, ewe age at birth, ewe weight at birth and birth weight as covariate. For statistical analysis we used the GENMOD procedure contained in SAS. Mean values for litter size and weaning rates were 1.36 and $68.18 \%$ respectively. The effect of ewe weight was significant about the litter size. There was no significant effect of birth season on the weaning rate. Correlations between productive and reproductive traits ranged from negative to positive low magnitude. The effects of ewe weight at birth and birth weight were important sources of variation for litter size and weaning rates, which reflects the need to consider these effects in models of genetic and phenotypic evaluation.

Keywords: birth season, fixed effects, hairless sheep, litter size 


\section{INTRODUÇÃO}

A demanda por carne ovina vem crescendo consideravelmente no Brasil. Para atender as exigências deste mercado e manter o crescimento do agronegócio, é necessário que a produção de cordeiros atenda a demanda e seja constante ao longo do ano. Nesse sentido, destaca-se que o desempenho reprodutivo do rebanho e a taxa de crescimento dos cordeiros estão entre os componentes responsáveis pelo sucesso do sistema de produção (MACEDO et al., 2003).

O comportamento reprodutivo dos animais em diferentes ambientes, bem como as técnicas de manejo reprodutivo apropriadas aos diferentes modelos físicos de exploração tem lugar de destaque no sistema de exploração.

A prolificidade possui grande contribuição relacionada com o ganho genético anual nos rebanhos em que se pratica seleção. Menores índices de prolificidade associados a baixas taxas de desmame implicam em menor número de cordeiros desmamados por matriz por ano, o que dificulta a reposição do plantel, diminui a pressão de seleção e aumenta o intervalo de geração, além de diminuir a taxa de desfrute do rebanho.

A literatura nacional ainda é incipiente no que diz respeito a estudos de algumas características reprodutivas de ovinos. Os escassos artigos verificados (SILVA \& ARAÚJO, 2000; MIRANDA \& MCMANUS 2000; ALVES et al. 2006) buscaram avaliar a influência de alguns efeitos do ambiente sobre a prolificidade e a taxa de desmame, empregando modelos que pressupõem distribuição normal para as características, quando, na prática, isso não ocorre, uma vez que estas apresentam distribuição discreta com respostas dicotômicas cuja magnitude só pode ser expressa em valores inteiros, sem frações.

Vale ressaltar que a analise considerando o tipo de distribuição dos dados é fator determinante para a correta inferência estatística. Desta forma, objetivou-se estudar a influência dos efeitos ambientais sobre a prolificidade e a taxa de desmame em fêmeas de ovinos da raça Santa Inês, empregando a teoria de modelos lineares generalizados.

\section{MATERIAL E MÉTODOS}

Os dados utilizados nesta pesquisa são originários do rebanho experimental de ovinos da raça Santa Inês, pertencentes ao Núcleo de seleção e conservação de recursos genéticos de ovinos deslanados para produção de carne no Sul do Piauí, implantado nas dependências da Universidade Federal do Piauí, Campus Universitário Professora Cinobelina Elvas, em Bom Jesus-PI no período de 2008 a 2010.

O manejo alimentar foi elaborado de forma que nos períodos de escassez de alimento (período de estiagem, compreendido entre os meses de junho a outubro) indistintamente todos os animais receberam, no cocho, suplementação alimentar à base de capim elefante (Penisetum purpureum schum, cv. Napier), feno de leucena (Leucaena leucocephala) e ração concentrada a base de milho e soja, na proporção de 40/60 (concentrado volumoso). Durante o período chuvoso (compreendido entre os meses de novembro a maio) os animais permaneceram em pastagem composta por capim Andropogon gayanus Kunth, cv. Planaltina (CIAT621) e vegetação nativa. Durante todo o período de pesquisa os animais tiveram 
à disposição sal mineral e água a vontade.

Foram coletadas informações referentes à identificação da ovelha, do reprodutor, do cordeiro, data da cobertura da ovelha, data do nascimento do cordeiro, sexo, tipo de nascimento dos cordeiros, peso ao nascer e número de animais desmamados por ovelha.

Os efeitos ambientais estudados sobre as características reprodutivas foram: ano de cobertura (2008, 2009 e 2010); Período de cobertura e nascimento (Período chuvoso - novembro a maio e período seco - junho a outubro); ano de nascimento (2009 e 2010); tipo de nascimento (simples e duplo); sexo da cria (macho e fêmea); idade da mãe ao parto (agrupada em classes, onde fêmeas com idade menor ou igual a dois anos compõe a classe um, fêmeas com idade maior que dois e menor ou igual a três anos compõe a classe dois, fêmeas com idade maior que três e menor ou igual a quatro anos compões a classe três e fêmeas com idade maior que quatro anos formam a classe quatro); peso da mãe ao parto (agrupada também em classes, onde fêmeas com peso menor ou igual a quarenta quilos formam a classe um e fêmeas com peso maior que quarenta quilos a classe dois); e peso ao nascer das crias como covariável linear e quadrática.

A prolificidade (PR) foi obtida pela relação do número de cordeiros nascidos por ovelha por parto. A taxa de desmame (TD) foi obtida pela atribuição de valores, sendo atribuído 0 para o animal nascido e não desmamado e 1 para o animal que foi desmamado. As características de prolificidade e taxa de desmame foram expressas, respectivamente, como simples ou duplos e desmamado ou não desmamado, esses dados são provenientes de uma distribuição discreta, e neste caso com duas classes, com uma probabilidade de ocorrência de cada classe, simples ou duplo e desmamado ou não desmamado. Desta forma, a análise desse tipo de informação objetiva modelar a probabilidade de ocorrência de cada classe em função de variáveis explicativas (efeitos de ambiente).

Inicialmente, o arquivo continha 753 registros. Para gerar o arquivo utilizado nas análises, foram feitas restrições, permanecendo no arquivo animais com pai e mãe conhecidos, ovelhas com, no máximo, quatro parições e grupos de contemporâneos com, no mínimo, cinco animais, restando 125 registros para as análises. Para realização da análise de consistência dos dados e a verificação dos efeitos de ambiente que podem influenciar a prolificidade, foram utilizados os procedimentos MEANS e GENMOD, respectivamente, do programa SAS (STATISTICAL ANALYSES SYSTEM, 1999). O GENMOD ajusta modelos lineares generalizados pelo método da máxima verossimilhança, utilizando uma função de ligação apropriada à probabilidade de distribuição. Utilizou-se a função de distribuição binomial e a função de ligação logística. O teste de $\chi^{2}$ de Pearson foi utilizado para verificar o ajuste da função de ligação aos dados. Dentro do procedimento GENMOD, utilizou-se a opção ESTIMATE, que calcula o logaritmo da razão de chances, in $\left[P_{i} / 1-P_{i}\right]$, em que $P_{i}$ representa a probabilidade de o parto ser duplo ou do animal ser desmamado e $1-P_{i}$ a probabilidade de ser simples ou do animal não ser desmamado. Para que os coeficientes estimados refletissem o efeito de o parto ser duplo, em relação ao simples e do animal ser desmamado, em relação a não desmamado, calculouse a exponencial do logaritmo da razão de chances, por meio da opção "EXP”, também disponível no procedimento mencionado, cuja resposta representa o 
número de vezes que a classe de parto duplo teria mais chances de ocorrer que a classe de parto simples, bem como o número de vezes que a classe de animais desmamados teria mais chances de ocorrer que a classe dos não desmamados.

As probabilidades de ocorrência de partos duplos e ocorrência de desmames foram calculadas como segue: $P_{i}=e / 1+e$, sendo:

$e=\mu+A C+E C+C I+C P, \quad$ para

partos duplos; e

$e=\mu+A N+E N+S R+T N+C l+C P+\sum_{i=1}^{2} \beta i(P N)^{2}$

, para ocorrência de desmame, em que: $\mu$ é uma constante inerente a todas as observações; $A C$ é o efeito de ano de cobertura (2008 a 2010); EC é o efeito de Período de cobertura (seco e chuvoso); Cl é a classe de idade da mãe ao parto; e $C P$ é a classe de peso da mãe ao parto; $A N$ é o efeito do ano de nascimento do cordeiro; $E N$ é o efeito de Período de nascimento do cordeiro; $S X$ é o efeito de sexo do cordeiro; $T N$ é o efeito de tipo de nascimento; $\beta_{i}$ são coeficientes de regressão do peso ao nascer dos cordeiros (linear e quadrático) sobre a taxa de desmame. A significância dos efeitos considerados foi testada pela estatística da razão de verossimilhança a 5\% de probabilidade.

\section{RESULTADOS E DISCUSSÃO}

O valor médio da prolificidade observado no rebanho foi de $1,36 \pm 0,48$. Observou-se taxa de desmame de 68,18\%. Silva \& Araújo (2000) observaram taxa de desmame de $70 \%$ para animais da raça Crioula e 38\% para animais mestiços Santa Inês, assim pode-se dizer que se trata de características que também sofre influência da raça.

As médias de peso ao nascer e peso ao desmame foram 3,24 $\pm 0,95$ e $10,44 \pm 4,15 \mathrm{~kg}$ respectivamente, o que estão próximas às relatadas na literatura (SILVA \& ARAÚJO, 2000; BARROS et al., 2004).

O efeito do peso da mãe ao parto foi significativo sobre a prolificidade $(\mathrm{P}<0,05)$. Como a metodologia utilizada permite calcular a probabilidade de ocorrência de partos múltiplos, considerando o efeito significativo incluído no modelo de análise, avaliouse o comportamento das probabilidades em função do peso da mãe ao parto. As chances de ocorrência de partos múltiplos observada para as classes de peso da mãe ao parto indicam que fêmeas com mais de $40 \mathrm{~kg}$ de peso vivo apresentaram 3,49 vezes mais chances de ter parto múltiplo que as fêmeas com menos de $40 \mathrm{~kg}$ de peso vivo. Silva \& Araújo (2000), empregando a metodologia de quadrados mínimos, onde se considera que a prolificidade apresenta distribuição normal, observaram efeito significativo apenas para $\mathrm{o}$ ano de parição.

A significância do peso da mãe ao parto sobre a prolificidade pode ser explicada pelo fato da prolificidade ser uma característica que está relacionada com o número e a qualidade de oócitos produzidos pela ovelha em cada ciclo estral, sendo que este número e a qualidade dependem da condição corporal da fêmea.

Para os efeitos de idade da mãe ao parto e período de cobertura não foram observadas diferenças significativas $(\mathrm{P}>0,05)$ sobre a prolificidade. Contrastando com esses resultados, Silva \& Araújo (2000) observaram que a prolificidade aumenta de acordo com a idade da ovelha, sendo as ovelhas com idade acima de 3,5 e 4,5 anos, 
respectivamente, as mais prolíferas. Em trabalho realizado adotando-se metodologia semelhante Santos et al. (2013) observaram as chances de ocorrências de partos múltiplos tendem a aumentar com o aumento da idade da matriz, ou seja, a medida que a fêmea tornou-se anatômica e fisiologicamente mais preparada.

Sarmento et al. (2010), ao estudarem a prolificidade de caprinos mestiços leiteiros no semiárido nordestino revelaram que os efeitos de mês de cobertura, idade da fêmea (ordem), idade ao primeiro parto, intervalo de partos e peso da mãe à cobertura foram significativos sobre a prolificidade $(\mathrm{P}<0,05)$, e afirmaram que fêmeas mais velhas, com maior intervalo de partos e maior idade ao primeiro parto tenderam a ter maior prolificidade, o que indica que estes efeitos devem ser considerados no estudo desta característica.

No que se refere à característica taxa de desmame, observou-se que o período de nascimento exerce efeito significativo $(\mathrm{P}<0,05)$ sobre esta, sendo que os animais nascidos no período seco apresentaram maior taxa de desmame que os nascidos no período chuvoso. Vale ressaltar que a taxa de desmame pode ser influenciada diretamente pelo peso ao nascer, pelos cuidados maternos pré-desmame, pela produção de leite da ovelha, pelo manejo sanitário e pelos cuidados em geral com o cordeiro do nascimento à desmama, sendo que os dois primeiros fatores podem ser influenciados diretamente da condição corporal da ovelha no momento do parto até o desmame.

Neste estudo, foi calculada a probabilidade de desmame dos animais, considerando o efeito significativo incluído no modelo de análise, onde foi avaliado o comportamento das probabilidades do período de nascimento em função do peso ao nascer dos cordeiros. Verificou-se que os animais nascidos no período seco apresentaram 4,85 vezes mais chances de serem desmamados que os animais nascidos no período chuvoso (Figura 1), visto que nos meses chuvosos ocorre maior incidência de verminoses que nas épocas secas. Além disso, o manejo sanitário adotado neste período pode ter sido ineficiente e contribuído para baixa taxa de desmame.

Independente do período de nascimento, observou-se que a probabilidade de desmame aumentou em função do aumento do peso ao nascimento (Figura 1). Este resultado confirma a importância do manejo nutricional durante $o$ período de gestação das fêmeas, pois cordeiros nascidos com bons pesos aumentam as chances de sobrevivência na desmama. Entretanto, o aumento indiscriminado do peso ao nascer deve ser visto com cautela, dado a possibilidade de distopia genital ao parto.

De acordo com Alves et al. (2006), existem evidências que a época seca favorece a saúde dos animais quando do nascimento, haja vista a ausência de umidade, o que proporciona ambiente favorável ao recém-nascido com a diminuição da incidência de doenças e reduzindo, consequentemente, a mortalidade nos primeiros dias de vida dos cordeiros.

Com a modelagem da variável resposta e adotando-se a distribuição adequada, observou-se que animais com maior peso ao nascer apresentaram maior probabilidade de desmame pela estatística da razão de verossimilhança $(\mathrm{P}<0,05)$ (Figura 1). Assim, animais que nasceram com maior peso, normalmente apresentaram maiores taxas de desmame, por outro lado, cordeiros nascidos pequenos e débeis, normalmente, têm menores possibilidades de 
Rev. Bras. Saúde Prod. Anim., Salvador, v.15, n.1, p.xxx-xxx jan./mar., 2014 http://www.rbspa.ufba.br ISSN 15199940

sobrevivência, em decorrência à dificuldade de procurar alimento, proporcionando menores taxas de desmame. Este fato evidencia a importância do peso ao nascer sobre a sobrevivência dos animais até 0 desmame e por toda sua vida.

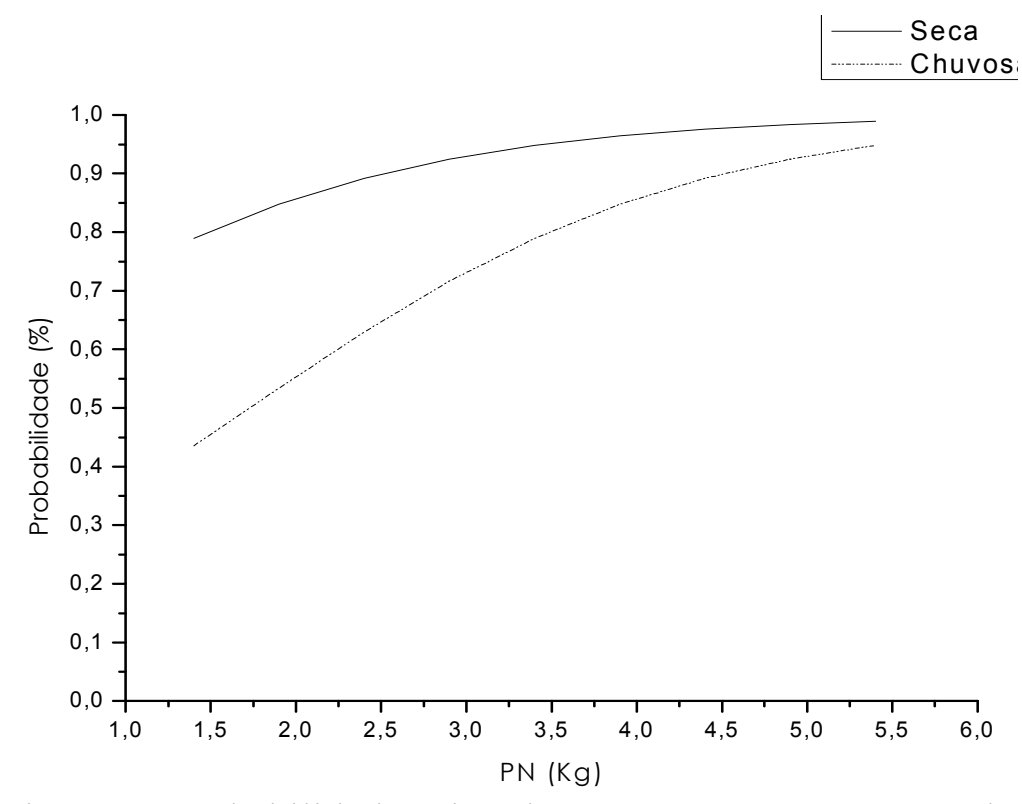

Figura 1. Probabilidade de desmame nas estações de nascimento seca e chuvosa em função do peso ao nascer $(\mathrm{PN})$ de ovinos da raça Santa Inês do Suldo Estado do Piauí

No que concerne os efeitos de peso da mãe ao parto, idade da mãe ao parto, ano de nascimento da cria, tipo de nascimento e sexo não foram significativos $(\mathrm{P}>0,05)$ sobre a taxa de desmame. Estes resultados diferem dos observados por Miranda \& McManus (2000), em que a taxa de desmame de cordeiros da raça Bergamácia foi influenciada $(\mathrm{P}<0,01)$ pelo peso ao nascer, tipo de nascimento, mês de nascimento e pelo ano de nascimento, atingindo $12 \% \mathrm{em}$ ano de seca rigorosa, o que gerou $88 \%$ de mortalidade. No entanto, destaca-se que a metodologia utilizada pelos mesmos aborda a teoria dos quadrados mínimos, que considera a taxa de desmame uma característica com distribuição normal, quando na verdade esta possui distribuição discreta.

As correlações de prolificidade com o peso ao nascer, taxa de desmame e peso aos 56 dias de idade variaram de positiva a negativa com baixa magnitude (Tabela 1). Isso confirma que os animais nascidos de partos múltiplos apresentaram menor peso aos 56 dias, consequente, menor taxa de desmame.

Sabe-se que quando o número de fetos presentes no útero materno aumenta, aumenta também a demanda nutricional e o espaço físico uterino torna-se limitado ao crescimento, o que pode influenciar o peso ao nascer dos cordeiros, resultado final do crescimento fetal, que pode ser explicado pela correlação negativa entre prolificidade e peso ao nascer (Tabela 1). 
Rev. Bras. Saúde Prod. Anim., Salvador, v.15, n.1, p.xxx-xxx jan./mar., 2014 http://www.rbspa.ufba.br ISSN 15199940

Tabela 1. Correlações entre características produtivas e reprodutivas de ovinos da raça Santa Inês criados sul do estado do Piauí

\begin{tabular}{lcccc}
\hline Características & IP & TD & PN & P56 \\
\hline PR & $0,13^{*}$ & $-0,16^{*}$ & $-0,20^{*}$ & $-0,38^{*}$ \\
IP & - & $-0,45^{*}$ & $-0,10^{*}$ & $-0,38^{*}$ \\
TD & - & - & $0,06^{*}$ & $-0,28^{\text {ns }}$ \\
PN & - & - & - & $0,37^{*}$ \\
\hline
\end{tabular}

*Significativo a 0,05 de probabilidade; ${ }^{\text {ns }}$ não significativo.

$\mathrm{IP}=$ intervalo de partos; $\mathrm{PN}=$ peso ao nascer; $\mathrm{P} 56$ = peso aos 56 dias de vida; $\mathrm{PR}=$ prolificidade; $\mathrm{TD}=$ taxa de desmame.

A correlação entre o peso ao nascer e o peso aos 56 dias (P56) de idade, que corresponde ao desmame dos animais, foi positiva, porém, com baixa magnitude, corroborando com os resultados observados por Silva \& Araújo (2000) que foi de 0,31.

Fêmeas que parem apenas uma única cria com baixo peso, estão sujeitas ao menor estresse ao parto e, consequentemente, têm menor predisposição a complicações pós-parto, facilitando a involução uterina e a recuperação de seu eixo hipotalâmicohipofisário-gonadal. Logo, a manifestação do período de estro pode ocorrer mais precocemente no pós-parto, possibilitando cobertura fértil mais precoce.

O peso da mãe ao parto é uma importante fonte de variação para prolificidade em ovinos. O peso ao nascer dos cordeiros é aspecto determinante para o aumento da taxa de desmame

\section{AGRADECIMENTOS}

A Universidade Federal do Piauí, pela disponibilidade dos animais e laboratório de informática para realização das análises estatísticas.

Ao Conselho Nacional de Desenvolvimento Científico e Tecnológico (CNPq), pelo apoio financeiro.

\section{REFERÊNCIAS}

ALVES, J.M.; McMANUS, C.; LUCCI, C.M.; CARNEIRO, H.C.R.;

DALLAGO, B.S.; CADAVID, V.G.; MARSIAJ, P.A.P.; LOUVANDINI, H. Season of birth and puberty in Santa Inês lambs. Revista Brasileira de Zootecnia, v.35, n.3, p.958-966, 2006. Supl.

BARROS, N.N.; VASCONCELOS, V.R. de.; LOBO, R.N.B. Growth traits of slaughter F1 lambs in northeast of Brazil. Pesquisa Agropecuária Brasileira, v.39, p.809-814, 2004.

MACEDO, F.A.F.; ZUNDT, M.; MEXIA, A.A. Parâmetros reprodutivos de matrizes ovinas, rebanho base para produção de cordeiros para abate.

Revista Brasileira de Reprodução Animal, v.27, p.127-133, 2003.

MIRANDA, R.M.; McMANUS, C. Performance of Bergamasca Sheep in the Brasília Region. Revista Brasileira de Zootecnia, v.29, p.1661-1666, 2000.

SANTOS, N.P.S.; SARMENTO, J.L.R.; PIMENTA FILHO, E.C.; CAMPELO, J.E.G.; FIGUEIREDO FILHO, L.A.S.; SOUSA JÚNIOR, S.C. Environmental and genetic aspects of litter size in goats using linear and threshold bayesian models. Arquivo Brasileiro de 
Rev. Bras. Saúde Prod. Anim., Salvador, v.15, n.1, p.xxx-xxx jan./mar., 2014 http://www.rbspa.ufba.br ISSN 15199940

Medicina Veterinária e Zootecnia, v.65, p.885-893, 2013.

SARMENTO, J.L.R.; PIMENTA

FILHO, E.C.; ABREU, U.G.P.;

RIBEIRO, M.N.; SOUSA, J. E.R. Litter size of crossbreed dairy goats in the northeastern semi-arid. Revista

Brasileira de Zootecnia, v.39, p.14711476, 2010.

SILVA, F.L.R.; ARAÚJO, A.M.

Reproductive Performance of Crossbred Santa Inês Sheep in Ceará State.

Revista Brasileira de Zootecnia, v.29, p.1712-1720, 2000.

STATISTICAL ANALYSES SYSTEM. Statistical analyses system: user's guide. Version 8. Cary, NC: SAS Institute. 1999.

Data de recebimento: 05/08/2013

Data de aprovação: 12/02/2014 